\title{
Ocorrência de febre amarela no Brasil: uma revisão integrativa da literatura (2014-2018)
}

\author{
Occurrence of yellow fever in Brazil: an integrative literature review (2014-2018)
}

Ocurrencia de fiebre amarilla en Brasil: una revisión integrativa de la literatura (2014-2018)

Tiago dos Santos Souza ${ }^{1}$, Ewerton Gomes de Almeida Coelho1, Thais Ranielle Souza de Oliveira ${ }^{*}$, Jéssica Caroline da Silva e Santos ${ }^{1}$, Pollyanna Barbosa Farias Barros¹.

\section{RESUMO}

Objetivo: Descrever através de uma revisão integrativa de literatura os aspectos da ocorrência da febre amarela no Brasil de 2014 a 2018. Métodos: Realizou-se um levantamento de artigos no período de 2014 a 2018, obtidos na EBSCO contendo as bases de dados: MEDLINE, Academic Search Elite, Dentistry \& Oral Sciences Source, Engineering Source, e demais bases como; National Library of Medicine National Health dos EUA (PubMed - NCBI), Biblioteca Virtual da Saúde (BVS), PlosOne, Scientific Eletronic Library Online (SCIELO), no mês de setembro de 2018, utilizando os descritores: Febre Amarela, prevenção, controle, epidemiologia, vacina e Brasil. Resultados: A amostra final foi composta por (12) artigos, (2) falavam sobre controle da febre amarela, (4) sobre aspectos epidemiológicos e (6) sobre imunização. Evidencia-se a relação entre incidência da febre amarela com fatores climáticos, diversidade de hospedeiros primatas não humanos e baixa cobertura vacinal. Conclusão: A cobertura vacinal deficiente em áreas de risco e a falhas nas informações e na descrição dos surtos pelos boletins epidemiológicos favorece o aumento da ocorrência desta doença no período analisado.

Palavras-chaves: Flavivírus, Prevenção, Cobertura vacinal, Epidemia.

\begin{abstract}
Objective: To describe, through an integrative literature review, aspects of the occurrence of yellow fever in Brazil from 2014 to 2018. Methods: A survey of articles was carried out in the period from 2014 to 2018, obtained in EBSCO containing the databases MEDLINE , Academic Search Elite, Dentistry \& Oral Sciences Source, Engineering Source, and other such bases; National Library of Medicine of the USA (PubMed - NCBI), Virtual Health Library (VHL), PlosOne, Scientific Eletronic Library Online (SCIELO), in September 2018, using the descriptors: Yellow Fever, prevention, epidemiology, vaccine, and Brazil. Results: The final sample consisted of (12) articles, (2) they talked about yellow fever control, (4) epidemiological aspects and (6) immunization. The relationship between yellow fever incidence and climatic factors, diversity of non-human primate hosts and low vaccination coverage is evidenced. Conclusion: Deficient vaccination coverage in areas of risk and failure of information and outbreak description by epidemiological bulletins favors the increase of disease occurrence in the period analyzed.
\end{abstract}

Keywords: Flavivirus, Prevention, Vaccination coverage, Epidemics.

${ }^{1}$ Centro Universitário Unieuro, Brasília -DF. *E-mail: thaisranielle@gmail.com

SUBMETIDO EM: 5/2019

ACEITO EM: 6/2019

PUBLICADO EM: 7/2019

REAS/EJCH | Vol.Sup.28 | e896 | DOI: https://doi.org/10.25248/reas.e896.2019 Página 1 de 10 


\section{RESUMEN}

Objetivo: Describir a través de una revisión integrativa de literatura los aspectos de la ocurrencia de la fiebre amarilla en Brasil de 2014 a 2018. Métodos: Se realizó un levantamiento de artículos en el período de 2014 a 2018, obtenidos en la EBSCO conteniendo las bases de datos: MEDLINE, Academic Search Elite, Odontología y Oral Ciencias Source, Engineering Source, y demás bases como, En el mes de septiembre de 2018, utilizando los descriptores: Fiebre Amarilla, prevención, control, fecundidad, epidemiología, vacuna y Brasil. Resultados: La muestra final fue compuesta por (12) artículos, (2) hablaban sobre control de la fiebre amarilla, (4) sobre aspectos epidemiológicos y (6) sobre inmunización. Se evidencia la relación entre incidencia de la fiebre amarilla con factores climáticos, diversidad de hospedadores primates no humanos y baja cobertura vacunal. Conclusión: La cobertura vacunal deficiente en áreas de riesgo y las fallas en las informaciones y en la descripción de los brotes por los boletines epidemiológicos favorece el aumento de la ocurrencia enfermedad en el período analizado.

Palabras claves:Flavivirus, Prevención, Cobertura vacunal, Epidemia.

\section{INTRODUÇÃO}

A febre amarela é uma doença infecciosa não contagiosa, transmitida aos humanos por meio da picada dos mosquitos infectados dos gêneros Aedes Aegypti e Haemagogus (CAPUTO LZ, et al., 2011; BRASILI R, et al., 2011; TAUIL PL, 2010). A doença pode apresentar dos ciclos de transmissão no urbano, o homem é fonte da infecção, sendo o mosquito Aedes o principal transmissor do vírus amarílico (BARATA RB e SAAD LDC, 2016). Já o ciclo silvestre mantém-se entre os primatas não humanos e o mosquito do gênero Haemagogus; o homem é acidentalmente contaminado quando, não imunizado, adentra áreas enzoóticas (TAUIL PL, 2010; BARATA RB e SAAD LDC, 2016).

As manifestações clínicas da febre amarela variam desde a forma assintomática até a mais grave da doença. Geralmente, na forma mais branda, o indivíduo apresenta hipertermia de leve a moderada, cefaleia e indisposição. Na forma moderada, ocorrem náuseas, mialgias, artralgias, e, pelo menos, um dos sintomas clássicos da febre amarela: hematêmese, icterícia e oligúria. Enquanto no quadro mais grave, o indivíduo apresenta esses três sintomas clássicos (CAPUTO LZ, et al., 2011; BRASILI, et al., 2011). A letalidade da febre amarela varia de $5 \%$ a $10 \%$, podendo chegar a $60 \%$ nas formas mais graves (BRASILI R, et al., 2011).

É uma doença caracterizada como endêmica, que apresenta grande impacto na saúde pública e que, apesar da disponibilidade da vacina antiamarílica, nos últimos vinte anos, a doença vem ressurgindo em países da África e nas Américas (BRASILI R, et al., 2011). Trinta e quatro países da África e treze das Américas Central e Sul são considerados endêmicos (WHO, 2016). No Brasil entre julho de 2017 e março de 2018, foram notificados 4.102 casos suspeitos de febre amarela no país, sendo confirmados 1.098 casos, e 340 desses casos confirmados evoluíram para óbito, 2.150 foram descartados e 854 ainda estavam sendo investigados (BRASIL, 2018).

A região Amazônica brasileira é considerada uma área endêmica; entretanto, outras regiões também vêm apresentando surtos esporádicos, em especial nas regiões do Centro-Oeste e Sudeste. A região Sudeste notificou no período entre 2016 e 2018 mais de 1.900 casos de febre amarela humana (ALVES PA, et al., 2018). O estado de São Paulo registrou no período de janeiro de 2018 até aquele presente momento 3028 casos suspeitos de Febre Amarela, sendo 537 casos confirmados (SES - SP, 2018). Já no estado de Minas Gerais foram confirmados 528 casos de febre amarela entre junho de 2017 a junho de 2018 (SES-MG, 2018).

A prevenção da febre amarela é realizada por meio da imunização e tem sido a grande aliada do Brasil no combate à doença. A vacina antiamarílica possibilitou o controle e a redução dos níveis de transmissão da doença (COSTA ZGA, et al., 2011). A imunização contra a febre amarela é realizada no Brasil desde 1937; a vacina contém vírus vivo atenuado e é produzida pelo Laboratório Bio-Manguinhos, da Fundação Oswaldo Cruz (CAPUTO LZ, et al., 2011; TAUIL PL, 2010). A vacina 17D é realizada em dose única e é considerada 
segura e muito eficaz pois confere proteção de mais de $95 \%$ dos receptores em até 30 dias após a sua aplicação (BARRETT ADT, et al., 2017).

Atualmente, o Ministério da Saúde disponibiliza a vacina para crianças a partir de nove meses e para adultos até os 59 anos; após essa idade é recomendável que seja feita a avaliação médica de risco benefício (BRASIL, 2018). A OMS recomenda a vacinação para todas as crianças a partir dos 6 meses de idade que vivem ou visitam áreas endêmicas (CAPUTO LZ, et al., 2011). O Ministério da Saúde aconselha aos viajantes com destinos a áreas endêmicas que a imunização seja realizada 10 dias antes (CAPUTO LZ, et al., 2011; ARAÚJO FAA, et al., 2011).

Diante do exposto anteriormente, da importância da atualização e produção de informação sobre a doença; o presente trabalho objetivou descrever a ocorrência da febre amarela no Brasil, no período entre 2014 a 2018, por meio de uma revisão integrativa da literatura.

\section{METODOLOGIA}

O presente estudo trata da revisão integrativa de literatura sobre a situação da ocorrência de febre amarela no Brasil nos últimos cinco anos. Foram definidos tanto o tema como a pergunta norteadora com a busca das definições e conhecimentos teóricos prévios para a formulação de uma questão para pesquisa, que apresentasse relevância na área escolhida.

Para a elaboração do estudo, formulou-se a seguinte pergunta norteadora: Quais os fatores que contribuem para a ocorrência da febre amarela no Brasil?

Realizou-se um levantamento de artigos no período de 2014 a 2018, obtidos na EBSCO contendo as bases de dados: MEDLINE, Academic Search Elite, Dentistry \& Oral Sciences Source, Engineering Source, e demais bases como; National Library of Medicine National Health dos EUA (PubMed - NCBI), Biblioteca Virtual da Saúde (BVS), PlosOne, Scientific Eletronic Library Online (SCIELO), no mês de setembro de 2018, utilizando os descritores: Febre Amarela, prevenção, controle, epidemiologia, vacina e Brasil e seus correspondentes em inglês "Yellow Fever, prevention, control, epidemiology, vaccine e Brazil".

Foram utilizados os operadores booleanos AND, que fornecem a intercessão entre os descritores escolhidos, e NOT para excluir assuntos não relacionados com o tema.

\section{Análise dos dados}

Na primeira etapa da pesquisa, foram utilizados todos os descritores nas bases de dados tendo como produto um total de 17316 artigos, sendo 2275 (EBESCO), 919 (PlosOne), 5673 (PubMed), 293 (Scielo) e 8156 BVS. Após aplicar os filtros (ano de publicação, idioma, texto completo e formato artigo), obteve-se como resultado 1.839 documentos, sendo 445 (EBESCO), 424 (PlosOne), 822 (PubMed), 48 (Scielo) e 100 (BVS).

$\mathrm{Na}$ segunda etapa, aferindo títulos e resumos, foram selecionados os artigos que abordavam exclusivamente o tema Febre Amarela, respeitando os critérios de inclusão: publicações entre o período de 2014 a 2018, artigos disponíveis na íntegra, gratuitos, em português ou inglês e com conexão com o tema.

Foram excluídos os documentos que não atenderam aos critérios de inclusão. Essa etapa teve como resultado o total de 159 trabalhos, sendo 52 (EBESCO), 06 (PlosOne), 34 (PubMed), 24 (Scielo) e 43 (BVS).

$\mathrm{Na}$ terceira fase foi realizada uma filtragem dos artigos repetidos, sendo excluídos 107 artigos e incluídos 52 artigos.

A quarta etapa foi composta pela avaliação dos artigos incluídos em periódicos de acordo com a classificação Qualis CAPES (BARATA RCB, 2016) e como o fator de Impacto "impactor factor", utilizado pelas bases de dados internacionais.

Foram incluídos os artigos classificados entre A1 e B2 e que apresentavam Fator de impacto $\geq 1.0$. Após esse procedimento restaram 41 artigos (Figura 1). 
Figura 1 - Diagrama dos métodos de identificação adaptado de NETO JD, et al. (2015). Brasília -DF, 2018.
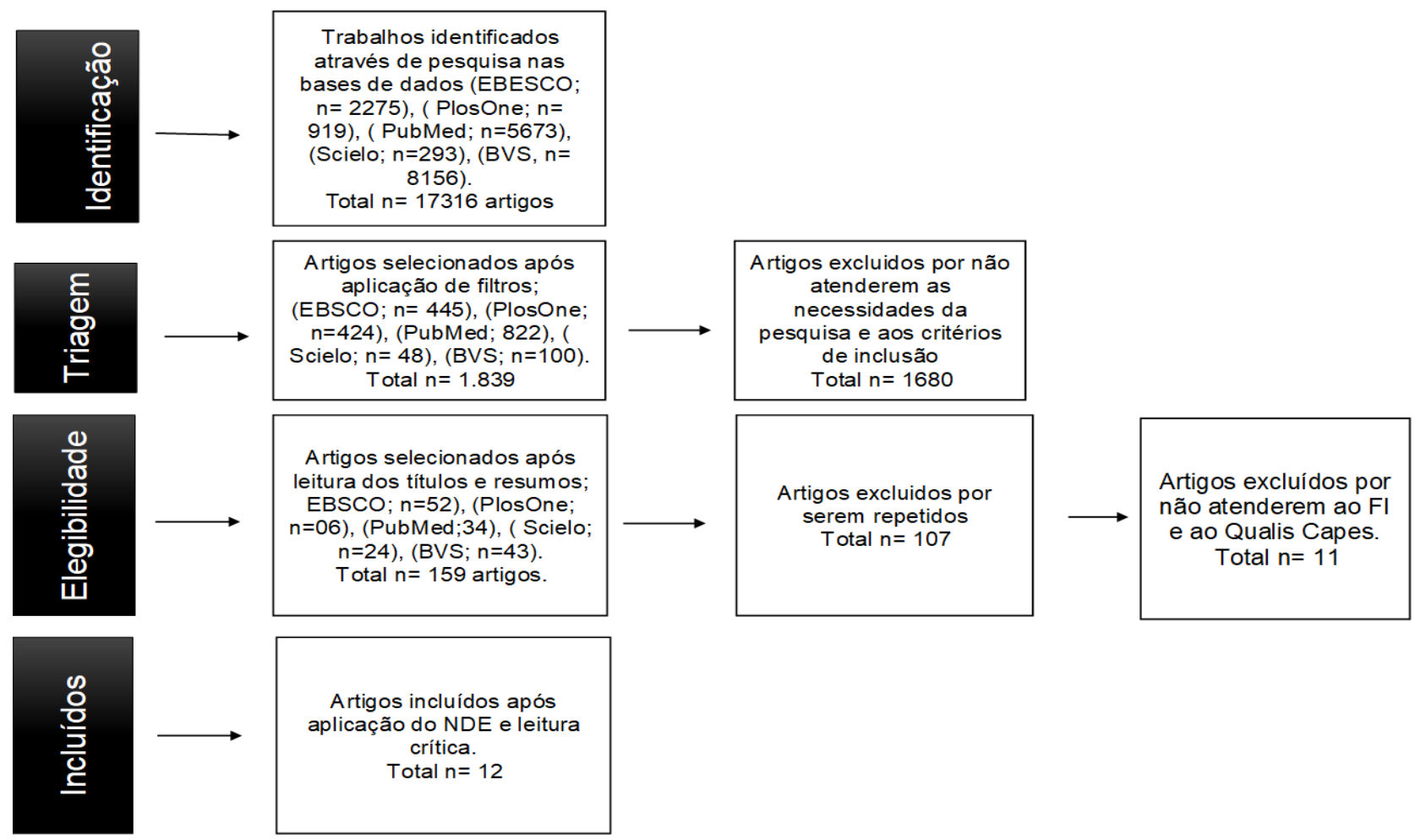

Fonte: Dados da pesquisa, 2018.

A amostra final foi constituída por 12 artigos. Logo, uma análise crítica dos trabalhos selecionados, realizada também de forma independente, permitiu a verificação das seguintes informações: ano, local de publicação, objetivos, metodologia, resultados com foco nos temas: aspectos epidemiológicos da febre amarela; imunização contra febre amarela e controle da febre amarela (Figura 1 e Quadro 1).

Quadro 1 - Fator de impacto das revistas que publicaram os artigos, adaptado de Qualis CAPES, segundo a plataforma sucupira www.capes.gov.br e Nível de evidência segundo critérios estabelecidos pela Oxford Centre for Evidence- based Medicine. Brasília -DF, 2018.

\begin{tabular}{|c|c|c|c|c|c|}
\hline REVISTA & CAPES & ISSN & $\begin{array}{l}\text { NÍVEL DE } \\
\text { EVIDÊNCIA } \\
\end{array}$ & $\begin{array}{l}\text { FATOR DE } \\
\text { IMPACTO }\end{array}$ & $\begin{array}{c}\text { AREA DE } \\
\text { AVALIAÇÃo }\end{array}$ \\
\hline ANNALS OF INTERNAL MEDICINE & A1 & $\begin{array}{l}0003- \\
4819\end{array}$ & II A & 19.384 & MEDICINA I \\
\hline $\begin{array}{l}\text { MORBIDITY AND MORTALITY } \\
\text { WEEKLY REPORT (PRINT) }\end{array}$ & A1 & $\begin{array}{l}0149- \\
2195\end{array}$ & IA & 23.370 & MEDICINA I \\
\hline $\begin{array}{l}\text { LANCET. INFECTIOUS DISEASES } \\
\text { (PRINT) }\end{array}$ & A1 & $\begin{array}{r}1473- \\
3099 \\
\end{array}$ & II A & 6.61 & MEDICINA I \\
\hline JAMA (CHICAGO, ILL.) & A1 & $\begin{array}{l}0098- \\
7484\end{array}$ & II A & 47.661 & MEDICINA I \\
\hline $\begin{array}{l}\text { PLOS NEGLECTED TROPICAL } \\
\text { DISEASES (ONLINE) }\end{array}$ & A2 & $\begin{array}{l}1935- \\
2735\end{array}$ & $\begin{array}{c}\mathrm{II} A / \mathrm{III} A / \mathrm{II} \\
\mathrm{A}\end{array}$ & 4.45 & MEDICINA I \\
\hline $\begin{array}{l}\text { RCM. RAPID COMMUNICATIONS } \\
\text { IN MASS SPECTROMETRY }\end{array}$ & B1 & $\begin{array}{r}0951- \\
4198\end{array}$ & III A/ IIIA/ & 1.97 & MEDICINA I \\
\hline $\begin{array}{l}\text { REVISTA DO INSTITUTO DE } \\
\text { MEDICINA TROPICAL DE SÃO } \\
\text { PAULO }\end{array}$ & B2 & $\begin{array}{l}1678- \\
9946\end{array}$ & II A & 1.489 & MEDICINA I \\
\hline $\begin{array}{l}\text { CURRENT INFECTIOUS DISEASE } \\
\text { REPORTS (PRINT) }\end{array}$ & B2 & $\begin{array}{l}1523- \\
3847\end{array}$ & III A & 2.085 & MEDICINA I \\
\hline $\begin{array}{l}\text { MEMÓRIAS DO INSTITUTO } \\
\text { OSWALDO CRUZ }\end{array}$ & B2 & $\begin{array}{l}1678- \\
8060\end{array}$ & III A & 2.833 & MEDICINA I \\
\hline
\end{tabular}

Fonte: Os autores, 2018. 


\section{RESULTADOS e DISCUSSÃO}

Foram selecionados doze artigos, publicados no período entre 2014 a 2018, sendo oito artigos publicados na PubMed, dois na Scielo e dois na EBESCO. Para melhor entendimento, os artigos selecionados foram separados por temas: quatro artigos abordaram os aspectos epidemiológicos da febre amarela, dois artigos trataram sobre o controle da doença e os outros seis artigos descreveram o aspecto vacinal da febre amarela. Os artigos utilizados no presente estudos são recentes; dos doze artigos selecionados, cinco foram publicados no ano de 2018, seis artigos no ano de 2017 e apenas um artigo foi publicado em 2014 (Figura 2).

Figura 2 - Distribuição dos artigos conforme ano de publicação. Brasília -DF, 2018.

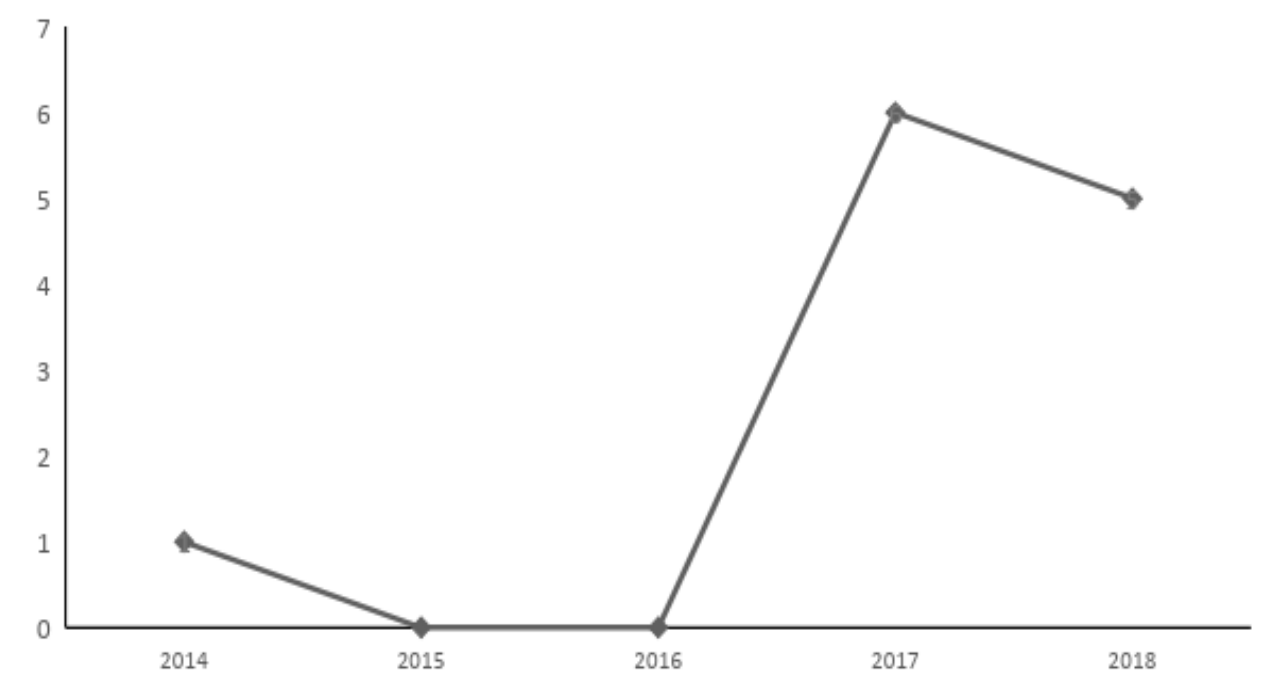

Fonte: Os autores, 2018.

\section{Aspectos Epidemiológicos da Febre Amarela}

Pesquisas apontam que fatores geoambientais como a chuva, altitude, temperatura, clima e a diversidades de hospedeiros primatas não humanos, estão intimamente ligados à presença de febre amarela (ALDIGHIERI S, et al., 2017; HOMMA A, et al., 2018).

No Brasil, atualmente, não há registros da febre amarela urbana (BRASILIA R, et al., 2011; TAUIL PL, 2010). Entretanto, existe certa preocupação por partes dos especialistas, em relação à reurbanização da febre amarela no Brasil. Essa preocupação refere-se ao ressurgimento do vírus da febre amarela que ocorre devido ao reaparecimento e à disseminação rápida de Aedes Aegypti no meio urbano (ALVES ABPS, et al., 2017).

Estudos comprovam que mudanças nas condições ambientais e climáticas favorecem a alta densidade de vetores silvestres e urbanos competentes e amplificadores primários dos hospedeiros vertebrados primatas não humanos (HOMMA A, et al., 2018; CHEN LH e HAMER DH, 2017).

O desmatamento, a ocupação descontrolada de áreas florestais, o grande número de primatas não humanos suscetíveis, a população sem imunização que reside em áreas peri-urbanas favorece a ocorrência de surtos da doença. No Brasil, ainda existem populações sem acesso a saneamento básico e à coleta de lixo nas áreas urbanas; tudo isso coopera com a proliferação do Aedes e aumenta o risco de possível reurbanização da febre amarela no Brasil (HOMMA A, et al., 2018).

Quanto ao Qualis, foram distribuídos da seguinte forma, artigos A1(4), artigos A2 (3), artigos B1 (2) e artigos B2 (3), todos com fator de impacto $\geq 1.0$. Em relação ao nível de evidência, os artigos selecionados apresentaram grau de recomendação IA, IIA ou IIIA (Tabela 2). 


\section{Revista Eletrônica Acervo Saúde / Electronic Journal Collection Health ｜ ISSN 2178-2091}

\begin{tabular}{|c|c|c|c|c|c|}
\hline $\begin{array}{c}\text { Base de } \\
\text { Dados }\end{array}$ & Título do artigo & Autor & Periódico (vol., $\mathbf{n}^{\circ}$, p., ano) & NDE & Considerações temáticas \\
\hline \multirow[t]{6}{*}{ PubMed } & $\begin{array}{l}\text { Aumento do risco de } \\
\text { infecções por febre } \\
\text { amarela entre viajantes } \\
\text { europeus não vacinados } \\
\text { devido a surto contínuo no } \\
\text { Brasil, julho de } 2017 \text { a } \\
\text { março de } 2018\end{array}$ & 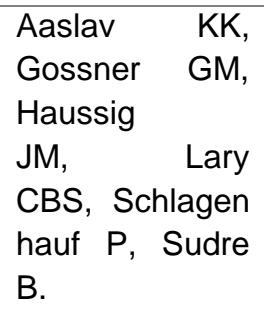 & $\begin{array}{l}\text { Rapid Communications in } \\
\text { Mass Spectrometry. v.16, no } \\
11 \text {, p. } 1-5,2018\end{array}$ & IIIA & $\begin{array}{l}\text { Apresenta aumento da transmissão nas regiões central e sudeste } \\
\text { relacionado a viagens entre europeus e sul-americanos não } \\
\text { vacinados. Sugere vacinação aos viajantes antes da viagem. }\end{array}$ \\
\hline & $\begin{array}{l}\text { Cobertura global de } \\
\text { vacina contra a febre } \\
\text { amarela de } 1970 \text { a 2016: } \\
\text { uma análise retrospectiva } \\
\text { ajustada }\end{array}$ & $\begin{array}{l}\text { Araújo VEM, et } \\
\text { al. }\end{array}$ & $\begin{array}{l}\text { Lancet Infect Dis v.17, } \\
\text { p.1109-1117, 2017. }\end{array}$ & IIA & $\begin{array}{l}\text { Aponta que houve melhora na cobertura vacinal, mas que ainda } \\
\text { é falha. Faz-se necessário o desenvolvimento de estratégias de } \\
\text { vacinação mais eficazes para evitar surto. }\end{array}$ \\
\hline & $\begin{array}{l}\text { Vacina 17D de Febre } \\
\text { Amarela Atenuada e Viva: } \\
\text { Uma Vacina Legada } \\
\text { Ainda Controlando Surtos } \\
\text { no Dia Moderno }\end{array}$ & $\begin{array}{l}\text { Asamene } \mathrm{N}, \\
\text { Adossa A, et al. }\end{array}$ & 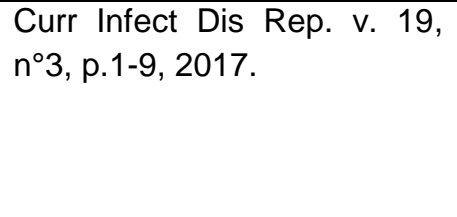 & IIIA & $\begin{array}{l}\text { Reforça a vacinação em dose única e a desnecessidade de } \\
\text { reforço a cada } 10 \text { anos. Questiona a eficácia da imunização e que } \\
\text { não existem estudos que comprovem a eficácia da vacina } \\
\text { fracionada. }\end{array}$ \\
\hline & $\begin{array}{l}\text { Desafios da vacinação no } \\
\text { confronto à ameaça } \\
\text { ressurgente da febre } \\
\text { amarela }\end{array}$ & $\begin{array}{l}\text { Chen } \\
\text { LH, Hamer DH }\end{array}$ & $\begin{array}{l}\text { JAMA, v. } 318, n^{\circ} 17, \text { p. } 1651- \\
52,2017 .\end{array}$ & IIA & $\begin{array}{l}\text { Revisão baseada em dados epidemiológicos de surtos de FA, } \\
\text { especialmente o ocorrido no Congo no período de dezembro de } \\
2015 \text { até } 2016 \text { reforça a prescindibilidade de dose de reforço. }\end{array}$ \\
\hline & $\begin{array}{l}\text { O risco internacional da } \\
\text { febre amarela se alastrou } \\
\text { do surto atual no Brasil, } \\
\text { de dezembro de } 2016 \text { a } \\
\text { maio de } 2017\end{array}$ & $\begin{array}{l}\text { Agua } \\
\text { R, } \quad \text { Cattarino } \\
\text { L, et al. }\end{array}$ & $\begin{array}{l}\text { Rapid Communications } \\
\text { EuroSurveill, v.22, n²8, p.1- } \\
4,2017 .\end{array}$ & IIIA & $\begin{array}{l}\text { Averiguou a probabilidade de um indivíduo viajante infectado com } \\
\text { FA no Brasil exportar essa doença para outro país e vice-versa. }\end{array}$ \\
\hline & $\begin{array}{l}\text { Surto Epizoótico de Vírus } \\
\text { da Febre Amarela e Risco } \\
\text { para Humanos Doença } \\
\text { em Salvador, Brasil }\end{array}$ & $\begin{array}{l}\text { Alves ABPS, } \\
\text { Campos GS. et } \\
\text { al }\end{array}$ & $\begin{array}{l}\text { Annals of Internal Medicine v. } \\
168, \text { n.4, p.301-302, } 2018 .\end{array}$ & IIA & $\begin{array}{l}\text { Por meio de testes com } 21 \text { primatas não humanos mortos no Rio } \\
\text { de Janeiro relatou que os mosquitos Aedes urbanos não estão } \\
\text { associados à transmissão do vírus da FA em seres humanos, } \\
\text { mas têm um risco considerável para a transmissão do mesmo. }\end{array}$ \\
\hline
\end{tabular}

REAS/EJCH | Vol.Sup.28 | e896 | DOI: https://doi.org/10.25248/reas.e896.2019 Página 6 de 10 


\section{Revista Eletrônica Acervo Saúde / Electronic Journal Collection Health ｜ ISSN 2178-2091}

\begin{tabular}{|c|c|c|c|c|c|}
\hline & $\begin{array}{l}\text { Padrões geográficos e } \\
\text { ambientais fatores } \\
\text { associados à presença da } \\
\text { febre amarela humana } \\
\text { nas Américas }\end{array}$ & $\begin{array}{ll}\frac{\text { Aldighieri }}{\text { Hamrick }} P N . \text { et } & \text { al } \\
\text { al } & \end{array}$ & $\begin{array}{l}\text { PLOS Neglected Tropical } \\
\text { Diseases, v. } 11, n^{\circ} 9, \text { p. } 1-28 \text {, } \\
2017 .\end{array}$ & IIIA & $\begin{array}{l}\text { Analisou a presença de FA por condado e sua forte ligação aos } \\
\text { fatores geoambientais. Esses fatores podem ajudar a prever e a } \\
\text { ajustar os limites de outras áreas de risco de interesse } \\
\text { epidemiológico. }\end{array}$ \\
\hline & $\begin{array}{l}\text { Surtos de Febre Amarela } \\
\text { em populações não } \\
\text { vacinadas, Brasil, 2008- } \\
2009\end{array}$ & $\begin{array}{l}\text { Andrade } \mathrm{MA} \text {, } \\
\text { Almeida } \mathrm{MAB} \text {. } \\
\text { et al }\end{array}$ & $\begin{array}{l}\text { PLOS Neglected Tropical } \\
\text { Diseases v.8, n³, p.1-9, } \\
2014 .\end{array}$ & IIIA & $\begin{array}{l}\text { Investigou casos de FA em população não vacinada e eventos } \\
\text { adversos em pessoas vacinadas. Aponta que não há } \\
\text { necessidade de reforço da vacina, apenas uma dose única }\end{array}$ \\
\hline \multirow[t]{2}{*}{ EBESCO } & $\begin{array}{l}\text { Persistência do vírus da } \\
\text { febre amarela fora da } \\
\text { Bacia Amazônica, } \\
\text { causando epidemias no } \\
\text { Sudeste do Brasil, de } \\
2016 \text { a } 2018\end{array}$ & Alves PA, et al & $\begin{array}{l}\text { PLOS Neglected } \text { Tropical } \\
\text { Diseasesv.12, } n^{\circ} 6, \quad \text { p.1-12, } \\
2018 .\end{array}$ & IIIA & $\begin{array}{l}\text { Aponta que a região Sudeste apresenta condições ecológicas e } \\
\text { climáticas adequadas para a manutenção do vírus durante a } \\
\text { epidemia e estações interepidêmicas, existindo riscos de ciclos e } \\
\text { epidemias enzoóticas de FA, fora da Bacia Amazônica no Brasil. }\end{array}$ \\
\hline & $\begin{array}{l}\text { Febre Amarela Fatal em } \\
\text { Viajantes para o Brasil, } \\
2018\end{array}$ & $\begin{array}{l}\text { Angelo } \\
\text { K, Caumes E. }\end{array}$ & $\begin{array}{l}\text { Morbidity and Mortality } \\
\text { Weekly Report } \\
\text { v.67, n¹1, p. } 340-341,2018 .\end{array}$ & IIA & $\begin{array}{l}\text { Aborda um grupo de } 10 \text { viajantes não vacinados que contraíram } \\
\text { FA no Brasil, no ano de } 2018 \text {. Recomenda que estes sejam } \\
\text { imunizados pelo menos } 10 \text { dias antes da viagem. }\end{array}$ \\
\hline \multirow[t]{2}{*}{ Scielo } & $\begin{array}{l}\text { O que esperar do surto de } \\
\text { febre amarela de } 2017 \text { no } \\
\text { Brasil? }\end{array}$ & $\begin{array}{l}\text { Angerami RN, } \\
\text { Luna EJA, } \\
\text { Rosseto EV. }\end{array}$ & $\begin{array}{l}\text { Revista do Instituto de } \\
\text { Medicina Tropical de São } \\
\text { Paulo, v.59, } \mathrm{n}^{\circ} 17, \quad \text { p.1-4, } \\
2017 \text {. }\end{array}$ & IIA & $\begin{array}{l}\text { Aponta deficiências dos boletins epidemiológicos em relação à } \\
\text { descrição dos surtos, às informações epidemiológicas e às } \\
\text { medidas de controle, o que dificulta o diagnóstico e medidas de } \\
\text { controle de vetores. }\end{array}$ \\
\hline & $\begin{array}{l}\text { Apelo urgente à ação: } \\
\text { evitar propagação e } \\
\text { reurbanização da febre } \\
\text { amarela no Brasil. }\end{array}$ & $\begin{array}{lr}\text { Homma } & \mathrm{A}, \\
\text { Martins } & \mathrm{RM}, \\
\text { Oliveira } & \mathrm{RL}, \\
\text { Possas C.. } & \end{array}$ & $\begin{array}{l}\text { Memórias Instituto Oswaldo } \\
\text { Cruz. v.113, } n^{\circ} 1, \text { p. 1-2, } 2018 .\end{array}$ & IIIA & $\begin{array}{l}\text { Relata vários fatores que cooperam para a propagação e } \\
\text { proliferação do Aedes, bem como para o aumento do risco de } \\
\text { reurbanização da FA no Brasil. }\end{array}$ \\
\hline
\end{tabular}

Fonte: Dados da pesquisa, 2018.

REAS/EJCH | Vol.Sup.28 | e896 | DOI: https://doi.org/10.25248/reas.e896.2019 Página 7 de 10 
Recentemente foram investigados casos humanos de febre amarela reportados à Organização PanAmericana de Saúde (OPAS) no período de 2000 a 2014. Foram notificados 1.164 casos de febre amarela em oito dos 13 países das Américas considerados endêmicos para o vírus da febre amarela pela Organização Mundial da Saúde (OMS). Quase $83,8 \%$ desses casos estavam concentrados em três países: Peru, Brasil e Colômbia. O Brasil foi o segundo país com maior número de casos $(28,1 \%)$. A maioria dos casos notificados concentrava-se nos estados de Minas Gerais e Goiás. (ALDIGHIERI S, et al., 2017). Esse mesmo estudo apontou que a febre amarela estava significativamente associada à altitude, chuva, diversidade de hospedeiros primatas não humanos e temperatura (ALDIGHIERI S, et al., 2017).

\section{Imunização contra Febre Amarela}

No Brasil a vacinação contra a febre amarela é administrada, principalmente, na imunização infantil de rotina em crianças a partir de nove meses de idade, em campanhas preventivas de massa, em resposta a surtos que visam à faixa etária mais ampla, e à vacinação em viajantes para áreas de alto risco (ARAUJO VEM, et al., 2017).

A vacina 17D atenuada tem sido bastante eficaz na prevenção de surtos da febre amarela. A imunização é realizada em dose única, não sendo mais necessária dose de reforço a cada 10 anos, como era preconizado no Brasil, anteriormente (CHEN LH, et al., 2017; ANDRADE MA, et al., 2014; BARRETT ADT, 2017).

Uma recente pesquisa explorou a cobertura vacinal contra a febre amarela, no período de 1970 a 2016 e os dados foram obtidos por meio de relatórios da OMS e registros de prestadores de serviços de saúde. $O$ estudo aponta que houve melhora na cobertura vacinal desde 1970, porém a cobertura vacinal no Brasil ainda é falha. Para atingir a cobertura global de $80 \%$, proposta pela OMS, seria necessário vacinar entre 393,7 milhões e 472,9 milhões de pessoas a mais do que é vacinado hoje. O autor recomenda o desenvolvimento de estratégias de vacinação mais eficazes para evitar surtos (ARAUJO VEM, et al., 2017).

$\mathrm{Na}$ iminência de um grande surto da febre amarela ocorrido no período entre dezembro de 2015 a dezembro de 2016, ocorrido na República Democrática do Congo e em Angola, devido à falta de vacina para a população e com objetivo de não deixar a população sem cobertura vacinal, foram utilizadas doses fracionadas da vacina contra a febre amarela. O Canadá também foi outro país que adotou o esquema de doses fracionadas, no intuito de enfrentar a falta da vacina (BARRETT ADT, 2017; CHEN LH e HAMER DH, 2017).

O Brasil assim como os Estados Unidos não utiliza a vacina da febre amarela em doses fracionadas por não existirem estudos que comprovem sua eficácia (CHEN LH e HAMER DH, 2017).

Um grupo de dez viajantes contraiu febre amarela no Brasil no ano de 2018. Entre os dez viajantes internacionais diagnosticados com febre amarela adquirida no Brasil, oito contraíram a doença na llha Grande, uma ilha florestada no Rio de Janeiro, onde já tinha sido detectado, em fevereiro de 2018, um caso de febre amarela humana e um caso de febre amarela em um primata não humano. Todos os 10 viajantes que contraíram a doença não estavam imunizados (ANGELO K, et al., 2018).

Uma pesquisa realizada em áreas de risco para febre amarela no Brasil investigou detalhadamente casos confirmados de febre amarela no período de julho de 2017 a março de 2018. Entre os casos estão dez europeus não vacinados que visitaram o Brasil. A pesquisa apontou aumento da transmissão da FA nas regiões central e sudeste do Brasil como a causa da ocorrência de casos de febre amarela, relacionados a viagens entre viajantes europeus e sul-americanos não vacinados, que visitaram destinos turísticos populares onde o vírus circula intensamente (AASLAV KK, et al., 2018). Estudos recomendam que os viajantes que planejam visitar áreas onde o vírus circula intensamente sejam vacinados pelo menos dez dias antes da viagem (ANGELO K, et al., 2018; AASLAV KK, et al., 2018).

\section{Controle da Febre Amarela}

A erradicação da febre amarela urbana no Brasil e nas Américas foi alcançada graças à combinação de campanhas de vacinação contra febre amarela e do combate ao vetor Aedes aegypti (HOMMA A, et al., 2018). Porém ainda circula no Brasil o vírus da febre amarela silvestre, causando surtos em vários estados 
brasileiros. Foi registrado no ano 2017 um grande surto de febre amarela ocorrido em Minas Gerais. Nesse período, 901 casos suspeitos foram notificados, dos quais 708 (78,6\%) casos e $60,1 \%$ das mortes permaneceram sob investigação (ANGERAMI RN, et al., 2017).

O estado de Minas Gerais foi o responsável por mais de $90 \%$ dos casos suspeitos de febre amarela; entretanto, apresentou apenas $20 \%$ do diagnóstico final. Esse mesmo estudo relata que os boletins epidemiológicos divulgados são muito ruins em relação à descrição do surto, às informações epidemiológicas e às medidas de controle, e isso deixa muitas dúvidas sobre o que fazer e que medidas tomar para evitar a reincidência de novos surtos (ANGERAMI RN, et al., 2017).

Os fatores geoambientais como a chuva, o clima, a altitude e a diversidade de hospedeiros primatas não humanos contribuem para a ocorrência da febre amarela. Entretanto, a ocupação descontrolada de áreas florestais, os primatas não humanos suscetíveis e a população não vacinada que residem em áreas periurbanas também são apontados como cooperadores para ocorrência de surtos da febre amarela no Brasil (ALDIGHIERI S, et al., 2017; HOMMA A, et al., 2018). A incidência da febre amarela silvestre é sazonal, coincidindo com a estação chuvosa na área endêmica, quando há aumento da densidade dos transmissores (CAVALCANTE KRLJ e TAUIL PL, 2016).

O Brasil tenta controlar a propagação da febre amarela e prevenir ocorrência de novos surtos da doença, principalmente por meio da imunização da população brasileira (BARRETT ADT e COLLINS ND, 2017; HOMMA A, et al., 2018; ARAUJO VEM, et al., 2017). Entretanto, vacinar somente a população brasileira não é o bastante para controlar a ocorrência da doença no Brasil, pois viajantes internacionais não vacinados visitam o Brasil e tanto podem adquirir a febre amarela em nosso país e exportá-la para o seu país de origem, como podem importar a febre amarela de seu país para o Brasil durante as viagens turísticas (ANDRADE MA, et al., 2014; ANGELO K, et al., 2018; AASLAV KK, et al., 2018; AGUAS R, et al., 2017).

Viajantes com destinos a áreas endêmicas sejam vacinados pelo menos dez dias antes da viajem (ANGELO K, et al., 2018; AASLAV KK, et al., 2018). Estudos sugerem dose única da vacina contra febre amarela sem necessidade de reforço; porém, o Ministério da Saúde recomenda dose de "reforço" aos viajantes vacinados há mais de 10 anos, com destino ao Brasil, especialmente em áreas de risco para febre amarela (BARRETT ADT e COLLINS ND, 2017; CHEN LH e HAMER DH, 2017). A fraca cobertura vacinal contra a febre amarela é um fator de relevante importância na ocorrência de febre amarela no Brasil (HOMMA A, et al., 2018).

\section{CONCLUSÃO}

Com base nos estudos a pergunta norteadora foi respondida. $O$ aumento de casos de febre amarela silvestre e o risco de reintrodução da forma urbana da doença por causa da fraca cobertura vacinal evidenciada na população adulta e viajantes internacionais, nos faz refletir sobre a necessidade de conscientização e adoção da vacina como a forma mais segura de prevenção.

\section{REFERÊNCIAS}

1. AASLAV KK, GOSSNER GM, HAUSSIG JM, et al. Increased risk of yellow fever infections among unvaccinated European travellers due to ongoing outbreak in Brazil, July 2017 to March 2018. Revista Rapid Communications in Mass Spectrometry. 2018 mar. v.16, no 11: 1-5.

2. AGUAS R, CATTARINO L, CORI A, et al. The international risk of yellow fever has spread from the current outbreak in Brazil, from December 2016 to May 2017. Revista Rapid. Communications EuroSurveill. 2017 jun.v.22, n²8 :1-4.

3. ALDIGHIERI S, HAMRICK PN, LEONEL DG, et al. Geographic patterns and environmental factors associated with human yellow fever presence in the Americas. Revista PLOS Neglected Tropical Diseases. 2017 set. v.11, n9:1-28.

4. ALVES ABPS, CAMPOS GS, CARDOSO CW, et al. Epizootic Outbreak of Yellow Fever Virus and Risk for Human Disease in Salvador, Brazil. Revista Annals of Internal Medicine. 2017 nov. v. 168, n4:301-302.

5. ALVES PA, ADELINO TER, BERNARDES AFL, et al. Persistent circulation of Yellow fever virus in Southeast Brazil, from 2016 to 2018. PLOS Neglected Tropical Diseases. 2018 jun. 
6. ANDRADE MA, ALMEIDA MAB, COSTA ZGA, et al. Yellow Fever outbreaks in unvaccinated populations, Brazil, 2008-2009. Revista PLOS Neglected Tropical Diseases. 2014 mar. v.8, n`3: 1-9.

7. ANGELOK, CAUMES E, CETRON M, et al. Fatal Yellow Fever in Travelers to Brazil, 2018. Revista Morbidade e Mortality W Relatório Eekly. 2018 mar. v.67, n:11 340- 341.

8. ANGERAMI RN, LUNA EJA, ROSSETO EV. What to expect from the 2017 yellow fever outbreak in Brazil? Revista do Instituto de Medicina Tropical de São Paulo. 2017 fev. v.59, n:17 1-4.

9. ARAÚJO FAA, ELKHOURY ANMS, LEAL SG, et al. Febre amarela no Brasil: recomendações para a vigilância, prevenção e controle. Revista Epidemiologia e Serviços de Saúde. 2011 jan. Brasília, v.20, n¹: 101-106.

10. ARAÚJO VEM, BRADY OJ, BROWNE AJ, et al. Global yellow fever vaccination coverage from 1970 to 2016 : an adjusted retrospective analysis. Revista Lancet InfectDis. 2017 ago. v.17:1109-1117.

11. BARATA RB, SAAD LDC.Surtos de febre amarela no estado de São Paulo, 2000-2010. Revista Epidemiologia e Serviços de Saúde. Brasília. 2016, v. 25, n³: 531-540.

12. BARATA RCB. Dez coisas que você deveria saber sobre o Qualis. RBPG. 2016 jan/abr v. 13, $n^{\circ} 1$ :

13. BARRETT ADT, COLLINS ND. Live Attenuated Yellow Fever 17D Vaccine: A Legacy Vaccine Still Controlling Outbreaks in Modern Day. Rev. Currlnfect Dis Rep. 2017 mar. v. 19, n:3 1-9.

14. BRASIL. Ministério da Saúde. 2018 ago.

15. BRASIL. Ministério da Saúde. 2018 mar. Disponível em: http://portalms.saude.gov.br/noticias/agencia-saude/42857febre-amarela-ministerio-da-saude-atualiza-casos-no-pais-4.

16. BRASIL. Secretaria de Saúde do Distrito Federal. Calendário Vacinal. 2018 mar. Disponível em: http://www.saude.df.gov.br/imunizacao-e-epidemiologia/

17. BRASILI R, FORTALEZA CMCB, MAEDA M, MASCHERETTI, et al. Febre amarela silvestre reemergência de transmissão no estado de São Paulo, Brasil, 2009. Revista Saúde Pública. São Paulo: 2011. v.47, n5: 881-889.

18. CAPUTO LZ, FONSECA FLA, FONSECA ALA, et al. Histórico da Febre Amarela no Brasil e a importância da vacinação antiamarílica. Arquivos Brasileiros de Ciências da Saúde. São Paulo: 2011. v.36, n¹ :40-47.

19. CARVALHO R, SILVA MD, SOUZA MT. Revisão integrativa: o que é e como fazer. Einstein. 2010 ago. vol.8 n¹: 102-106

20. CAVALCANTE KRLJ, TAUIL PL. Características epidemiológicas da febre amarela no Brasil, 2000-2012*. Revista Epidemiologia e Serviços de Saúde. 2016 jan/mar. vol. 25, n:1 11-20.

21. CEBM: Oxford Centre for Evidence- based Medicine Levels of Evidence Grades of Recommendation (April 2001). University of Oxford.

22. CHEN LH, HAMER DH. Vaccination Challenges in Confronting the Resurgent Threat from Yellow Fever. Revista JAMA. 2017 nov. v. 318, n¹7:1651-52

23. COSTA CGA, GOMES AC, GUTIERREZ MFC, et al. Registro de Aedes albopictus em áreas epizoóticas de febre amarela das Regiões Sudeste e Sul do Brasil (Diptera: Culicidae) *. Revista Epidemiologia e Serviços de Saúde. 2008 jan. v.17, $n^{\circ} 171-76$.

24. COSTA ZGA, ELKHIURY ANM, FLANNERY B, et al. Evolução histórica da vigilância epidemiológica e do controle da febre amarela no Brasil. Revista Pan- Amaz. Saúde. 2011 fev. v.2, n¹:11-26.

25. GOVERNO DO ESTADO DE SÃO PAULO. Secretaria de Estado da Saúde Boletim Epidemiológico Febre Amarela 2018.

26. HOMMA A, MARTINS RM, OLIVEIRA RL, et al. Urgent call for action: avoiding spread and re-urbanisation of yellow fever in Brazil. Revista Memórias Instituto Oswaldo Cruz. 2018 jan. Rio de Janeiro, v.113, n¹: 1-2.

27. NETO JD, NAKAMURA AS, CORTEZ LER, et al. Doenças sexualmente transmissíveis em idosos: uma revisão sistemática. Revista Ciência Saúde Cole. 2017 jun. v.20, n¹2:3853-64.

28. SES - MG. Informe Epidemiológico da Febre Amarela, Minas Gerais. 2018 out. Disponível em: http://saude.mg.gov.br/component/gmg/story/10625-informe-epidemiologico-da-febre-amarela-21-06.

29. TAUIL PL. Aspectos críticos do controle da febre amarela no Brasil. Revista Saúde Pública. Brasília: 2010. v.44, n.16: 555-558.

30. VASCONCELOS PFC. Febre amarela: reflexões sobre a doença, as perspectivas para o século XXI e o risco da reurbanização. Revista Brasileira de Epidemiologia. 2002 vol.5, n³:244-258.

31. WORLD HEALTH ORGANIZATION. Febre amarela. 2016 maio. Disponível em: http://www.who.int/mediacentre/factsheets/fs100/pt/ 